\title{
MONOMIALS IN THE JONES PROJECTIONS
}

\author{
V.S. SUNDER
}

(Communicated by John B. Conway)

\begin{abstract}
It is shown that every monomial $e_{I}=e_{i_{1}} e_{i_{2}} \cdots e_{i_{n}}$ in the Jones projections (with parameter $\tau$ ) satisfies $e_{I}=\tau^{n(I) / 2} u_{I}$ where $u_{I}$ is a partial isometry and $n(I)$ is an integer for which an explicit formula is given.
\end{abstract}

Let $\left\{e_{n}: n \geq 0\right\}$ be the sequence of Jones projections associated to a fixed number $\left.\tau \in\left(0, \frac{1}{4}\right] \cup \frac{1}{4} \sec ^{2} \pi / n: n \geq 3\right\}$; thus each $e_{i}$ is an orthogonal projection and the following commutation relations hold:

$$
\begin{aligned}
& e_{i} e_{j}=e_{j} e_{i} \quad \text { if }|i-j|>1, \\
& e_{i} e_{i \pm 1} e_{i}=\tau e_{i}
\end{aligned}
$$

For any string $I=i_{1} i_{2} \cdots i_{n}$ of nonnegative integers, we shall write $e_{I}=e_{i_{1}} e_{i_{2}} \cdots$ $e_{i_{n}}$. With $I$ as above, we shall write $I \backslash\left\{i_{\tau}\right\}$ for the string $i_{1} \cdots i_{\tau-1} i_{\tau+1} \cdots i_{n}$. More generally, if a string $I$ is obtained by dropping some integers from a string $J$, we shall call $I$ a substring of $J$.

The relations (1) above are seen to bear a striking resemblance to the relations in the presentations of the Braid groups as well as the Hecke algebras. Both these connections have, as is well-known, been very fruitfully exploited by Jones, Ocneanu, Wenzl and several others. As is customary when dealing with generators and relations, it is often convenient to work with reduced words. (For the case of Coxeter groups, this is classical and may be found in several places; see, for instance, [1].) For the particular case in hand, we shall find it convenient to use the following description of reduced words, which may be found in [2] (Aside 4.1.4): If $J$ is any string, then there exists a string $I$ and an integer $l$ such that $e_{J}=\tau^{l} e_{I}$ and such that $I$ has the 'canonical form'

$$
\begin{aligned}
& I=j_{1}\left(j_{1}-1\right) \cdots k_{1} j_{2}\left(j_{2}-1\right) \cdots k_{2} \cdots j_{p}\left(j_{p}-1\right) \cdots k_{p} \\
& \text { with } k_{i}<k_{i+1} \text { and } j_{i}<j_{i+1} \quad \text { for } 1 \leq i<p .
\end{aligned}
$$

When $I$ is as in (2), we shall say that $I$ is in canonical form, with $p$ blocks, the $i$ th block of $I$ being the substring $j_{i}\left(j_{i}-1\right) \cdots k_{i}$, finally, we write $b(I)=\sum_{i=1}^{p}\left(j_{i}-k_{i}\right)$ and refer to $b(I)$ as the block-length of $I$.

Received by the editors November 12, 1986 and, in revised form, April 13, 1987.

1980 Mathematics Subject Classification (1985 Revision). Primary 46L10; Secondary 22D25.

Key words and phrases. Monomials in Jones projections, canonical form, partial isometry, square of norm. 
LEMMA 1. Let I be as in (2) above. Define

$$
\begin{aligned}
J= & j_{1} j_{2} \cdots j_{p}\left(j_{1}-1\right)\left(j_{1}-2\right) \\
& \cdots k_{1}\left(j_{2}-1\right)\left(j_{2}-2\right) \cdots k_{2} \cdots\left(j_{p}-1\right)\left(j_{p}-2\right) \cdots k_{p}, \\
K= & j_{1}\left(j_{1}-1\right) \cdots\left(k_{1}+1\right) j_{2}\left(j_{2}-1\right) \\
& \cdots\left(k_{2}+1\right) \cdots j_{p}\left(j_{p}-1\right) \cdots\left(k_{p}+1\right) k_{1} k_{2} \cdots k_{p}
\end{aligned}
$$

it being understood that if some $j_{i}=k_{i}$, the corresponding 'empty' string in $J$ and $K$ is said to be omitted; then $e_{I}=e_{J}=e_{K}$.

PROOF. This follows immediately from the commutation relations (1) and the inequalities in the definition of the canonical form (2).

PROPOSITION 1. If $I$ is any string, there exist an integer $n(I)$ and a partial isometry $u_{I}$ such that $e_{I}=\tau^{n(I) / 2} u_{I}$.

ProOF. The proof is by induction on $l(I)$, the length of the string $I$. The statement is obvious when $l(I)=1$. Suppose the statement is valid for any string $J$ with $l(J)<l(I)$.

We may clearly assume that $I$ is in canonical form and given by (2). If $j_{1}>k_{1}$, then $e_{I}^{*} e_{I}=\tau e_{J}^{*} e_{J}$ where $J=I \backslash\left\{j_{1}\right\}$; since $l(J)=l(I)-1$, the induction hypothesis settles this case. We may, thus, assume that $j_{1}=k_{1}$.

Next, if $k_{1}\left(=j_{1}\right)<k_{2}-1$, it follows from (1) that if $J=I \backslash\left\{k_{1}\right\}$, then $e_{k_{1}}$ commutes with $e_{J}$, so $e_{I}^{*} e_{I}=e_{k_{1}} e_{J}^{*} e_{J}=\tau^{n(J)} e_{k_{1}} u_{J}^{*} u_{J}$, and $e_{k_{1}} u_{J}^{*} u_{J}$ is a projection (being a product of commuting projections). Thus, we may also assume that $k_{2}=$ $k_{1}+1$.

Hence there exist indices $i\left(i=1\right.$ works) such that $k_{i+1}=k_{i}+1$. Let $r$ be the largest such index. Then $k_{r+1}<k_{i}-1$ for all $i>r+1$. It follows from Lemma 1 and the relations (1) that $e_{I}=e_{L}$ where

$$
\begin{aligned}
L= & j_{1} \cdots k_{1} \cdots j_{r-1} \cdots k_{r-1} j_{r} \\
& \cdots\left(k_{r}+1\right) j_{r+1} \cdots\left(k_{r+1}+1\right) j_{r+2} \cdots k_{r+2} \cdots j_{p} \cdots k_{p} k_{r} k_{r+1} ;
\end{aligned}
$$

then, $e_{I} e_{I}^{*}=e_{L} e_{L}^{*}=\tau e_{J} e_{J}^{*}$ where $J=L \backslash\left\{k_{r+1}\right\}$. By induction hypothesis, $e_{J} e_{J}^{*}=$ $\tau^{n(J)} q_{J}$ where $q_{J}=u_{J} u_{J}^{*}$ is a projection; hence $e_{I} e_{I}^{*}=\tau^{n(J)+1} q_{J}$, so that the proposition is valid for $I$, with $n(I)=n(J)+1$.

We turn now to the determination of $n(I)$ (as in Proposition 1). Notice that $\tau^{n(I)}=\left\|e_{I}\right\|^{2}$.

PROPOSITION 2. Let I be in canonical form and given by (2). Inductively define the integers $l_{1}, l_{2}, \ldots, l_{p}$ thus: $l_{1}=k_{1} ;$ if $1<i \leq p$ and $l_{i-1}$ has been defined, let

$$
l_{i}= \begin{cases}\min \left\{j_{i}, l_{i-1}+2\right\}, & \text { if } j_{i}>k_{i} \leq l_{i-1}+1, \\ k_{i}, & \text { otherwise. }\end{cases}
$$

Then $n(I)=b(I)+\#\left\{i: 1 \leq i<p, l_{i+1}=l_{i}+1\right\}$.

PROOF. The proof is by induction of $b(I)$. (Recall that $b(I)=\sum_{i=1}^{p}\left(j_{i}-k_{i}\right)$.)

If $b(I)=0$, then for each $i$, we have $j_{i}=k_{i}=l_{i}$. Thus $e_{I}=e_{l_{1}} e_{l_{2}} \cdots e_{l_{p}}$, and since $l_{1}<\cdots<l_{p}$, it is clear from (1) that $n(I)=\#\left\{i: 1 \leq i<p, l_{i+1}=l_{i}+1\right\}$.

Assume now that the proposition is valid for any string $J$ which is in canonical form and satisfies $b(J)<b(I)$, and that $b(I)>0$. The induction step will be 
complete once we can find a substring $J$ of $I$ such that

(i) $J$ is in canonical form;

(ii) $\left\|e_{I}\right\|^{2}=\tau\left\|e_{J}\right\|^{2}$ (so that $n(I)=n(J)+1$ );

(iii) $b(I)=b(J)+1$;

(iv) both $I$ and $J$ have the same associated $l_{i}$ 's.

We shall obtain $J$ by dropping from $I$ some member of some nontrivial block. Thus, suppose $1 \leq r \leq p, j_{r}>k_{r}$ and $j_{r} \geq m \geq k_{r}$ and $J=I \backslash\{m\}$. Since the $l_{i}$ 's are defined inductively, it is clear that $l_{i}(I)=l_{i}(J)$ for $i<r$. Further, if it is the case that $l_{r}(I)=l_{r}(J)$, it would then follow (from the fact that $l_{i}$ depends only on the $i$ th block and $\left.l_{i-1}\right)$ that also $l_{i}(I)=l_{i}(J)$ for $i>r$. So if $J$ is constructed in the manner described, we would only have to verify $l_{r}(I)=l_{r}(J)$ in order to establish (iv).

Let $s$ be the index of the first nontrivial block in $I$; i.e., $s=\min \{i: 1 \leq i \leq$ $\left.p, j_{i}>k_{i}\right\}$.

Case (i). $s=1$, or $s>1$ and $k_{s}-1>k_{s-1}\left(=j_{s-1}\right)$.

In this case, we let $J=I \backslash\left\{j_{s}\right\}$, and observe that (i) follows at once, as does (iii), while the commutation relations (1) ensure that $e_{I}^{*} e_{I}=\tau e_{J}^{*} e_{J}$ and hence (ii) follows. As for (iv), notice that $l_{i}(I)=l_{i}(J)=j_{i}$ for $i<s$; since $k_{s}>l_{s-1}+1$, we have $l_{s}(I)=l_{s}(J)=k_{s}$, and hence (iv) follows from the above remarks.

Case (ii). $s>1$ and $k_{s}-1=k_{s-1}\left(=j_{s-1}\right)$.

In this case, there exist indices $i$ (for instance, $i=s$ ) which correspond to nontrivial blocks and satisfy $k_{i}=k_{i-1}+1$. Let $r$ be the largest such index, and put $J=I \backslash\left\{k_{r}\right\}$.

If $r=p$, (i) is immediate; if $r<p$, then either $k_{r+1}=j_{r+1}>j_{r} \geq k_{r}+1$, or $j_{r+1}>k_{r+1}>k_{r}+1$; so, in any case $k_{r-1}+1=k_{r}<k_{i}-1$ for $i>r$. It follows at once that (i) (and clearly, also (iii)) is valid; also, the above inequalities ensure that $\left(e_{k_{r-1}} e_{k_{r}}\right.$ can be pulled to the extreme right in $e_{I}$ and that) $e_{I} e_{I}^{*}=\tau e_{J} e_{J}^{*}$ and hence (ii) holds. It remains only to establish $l_{r}(I)=l_{r}(J)$, and we do this by considering two cases.

Case (iia). $l_{r-1}=k_{r-1}$. (Note that $l_{r-1}(I)=l_{r-1}(J)$.)

Here, $k_{r}=l_{r-1}+1$ and also $j_{r}>k_{r}$ (by the definition of $r$ ) and hence,

$$
l_{r}(I)=\min \left\{j_{r}, l_{r-1}+2\right\}=k_{r}+1 .
$$

On the other hand, $k_{r}(J)=k_{r}+1>l_{r-1}(J)+1$ and hence $l_{r}(J)=k_{r}(J)=k_{r}+1$, as desired.

Case (iib). $l_{r-1}>k_{r-1}$. (Notice that $j_{i} \geq l_{i} \geq k_{i} \forall i$.)

Here, $j_{r}>k_{r}=k_{r-1}+1 \leq l_{r-1}$ and so $l_{r}(I)=\min \left\{j_{r}, l_{r-1}+2\right\}$.

On the other hand, $k_{r}(J)=k_{r}+1 \leq l_{r-1}+1$, and we must distinguish between the cases $j_{r}=k_{r}+1$ and $j_{r}>k_{r}+1$. If $j_{r}=k_{r}+1$, then $l_{r}(J)=k_{r}(J)=k_{r}+1$, while $j_{r} \leq l_{r-1}+1$ implies that

$$
l_{r}(I)=\min \left\{j_{r}, l_{r-1}+2\right\}=j_{r}=k_{r}+1=l_{r}(J) .
$$

If $j_{r}>k_{r}+1$, then

$$
l_{r}(J)=\min \left\{j_{r}, l_{r-1}+2\right\}=l_{r}(I),
$$

and the proof is complete.

EXAMPLES. (1) If $I=0214326543765$, then $b(I)=0+1+2+3+2=8$, and $l_{1}=0, l_{2}=2, l_{3}=4, l_{4}=6, l_{5}=7$; since there is only one pair of successive integers in the $l_{i}$ sequence, we find that $\left\|e_{I}\right\|^{2}=\tau^{9}$. 
(2) Let $n \geq 1$ and $I=n(n-1) \cdots 0(n+1) n \cdots 1 \cdots(2 n)(2 n-1) \cdots n$. In our notation, $p=n+1, j_{i}=n+i-1, k_{i}=i-1$ and $b(I)=(n+1) n$. It is easy to see, inductively, that $l_{i}=2(i-1)$ for each $i$, and hence $\left\|e_{I}\right\|^{2}=\tau^{n(n+1)}$. Also, a repeated application of Lemma 1 shows that

$$
\begin{aligned}
e_{I} & =e_{n} \cdots e_{1} e_{n+1} \cdots e_{2} \cdots e_{2 n} \cdots e_{n+1} e_{0} e_{1} \cdots e_{n} \\
& =e_{n} \cdots e_{2} e_{n+1} \cdots e_{3} \cdots e_{2 n} \cdots e_{n+2} e_{1} e_{2} \cdots e_{n+1} e_{0} e_{1} \cdots e_{n} \\
& =\cdots \\
& =e_{n} e_{n+1} \cdots e_{2 n} e_{n+1} e_{n+2} \cdots e_{2 n-1} \cdots e_{1} e_{2} \cdots e_{n+1} e_{0} e_{1} \cdots e_{n} \\
& =e_{I}^{*} .
\end{aligned}
$$

It follows that $\tau^{-n(n+1) / 2} e_{I}$ is a projection. It was shown in [3 and 4] that if $M_{-1} \subseteq$ $M_{0}$ is a pair of $\mathrm{II}_{1}$ factors with $\left[M_{0}: M_{-1}\right]=\tau^{-1}$, and if $M_{-1} \subseteq M_{0} \subseteq M_{1} \subseteq M_{2} \subseteq$ .. is the tower of $\mathrm{II}_{1}$ factors obtained by iterating the basic construction, so that $M_{n+1}=\left(M_{n} \cup\left\{e_{n}\right\}\right)^{\prime \prime}$, then for any $n \geq 1, M_{-1} \subseteq M_{n} \subset M_{2 n+1}$ 'is also a basic construction' and the projection in $M_{2 n+1}$ which implements the conditional expectation of $M_{n}$ onto $M_{-1}$ is precisely the ' $\tau^{-n(n+1) / 2} e_{I}$ ' of this example. See [4] for another proof of the fact that this is a projection. In fact, it was an examination of this 'example' that resulted in this short note.

\section{REFERENCES}

1. N. Bourbaki, Groupes et algèbres de Lie, vols. IV, V, VII, Hermann, Paris, 1968.

2. V. F. R. Jones, Index for subfactors, Invent. Math. 72 (1983), 1-25.

3. A. Ocneanu, $A$ Galois theory for operator algebras, preprint.

4. M. Pimsner and S. Popa, Iterating the basic construction, preprint.

Department of Mathematics, University of California, Los Angeles, CaliFORNIA 90024

Current address: Indian Statistical Institute, Bangalore Centre, Bangalore 560 059, India 